\title{
miR-190b promotes colorectal cancer progression through targeting forkhead box protein P2
}

\author{
QIANG ZHAO, CHENGKUI LIU, QING CUI, XUERONG LUAN, QINGFENG WANG and CHENGFU ZHOU
}

Department of Gastrointestinal Surgery, Zibo Central Hospital, Zibo, Shandong 255000, P.R. China

Received November 22, 2018; Accepted October 3, 2019

DOI: $10.3892 /$ etm.2019.8175

\begin{abstract}
RNA-190b (miR-190b) is abnormally expressed in multiple types of cancer, however, its role in colorectal cancer (CRC) is largely unknown. In the present study, it was demonstrated that miR-190b expression was upregulated in CRC cell lines compared with the normal epithelial colon cell line. Knockdown of miR-190b decreased proliferation, colony formation and invasion, and increased apoptosis of CRC cells. Furthermore, forkhead box protein P2 (FOXP2) was predicted as a target of miR-190b and further validated by luciferase activity reporter assay and western blotting. Rescue experiments showed that knockdown of FOXP2 reversed the inhibitory effects of miR-190b inhibitor on the behavior of the CRC cell lines. Taken together, the present study demonstrated the oncogenic role of miR-190b in $\mathrm{CRC}$ through regulation of FOXP2 expression.
\end{abstract}

\section{Introduction}

Colorectal cancer (CRC) is one of the most common malignancies worldwide, and results in $\sim 600,000$ deaths per year (1). The 5-year overall survival (OS) rate for patients with early-stage CRC is $\sim 90 \%$; however, in patients with advanced CRC OS is only $\sim 10 \%$ (2). Outcomes of patients with CRC may be improved by targeting key molecules associated with progression of CRC (3). Therefore, improving our understanding of carcinogenesis of CRC may assist in the design of novel molecular targeting strategies.

MicroRNAs (miRNAs) are single-stranded non-coding RNAs that can suppress gene expression primarily through binding to the 3'-untranslated regions (3'-UTRs) of target mRNAs (4). miRNAs are able to interact with multiple mRNAs, influencing various cellular functions (5). Studies have demonstrated that miR-190b is abnormally expressed in

Correspondence to: Dr Chengfu Zhou, Department of Gastrointestinal Surgery, Zibo Central Hospital, 54 Gongqingtuan West Road, Zhangdian, Zibo, Shandong 255000, P.R. China E-mail: chengfu_zhou@yeah.net

Key words: miR-190b, forkhead box protein P2, colorectal cancer, oncogene, progression multiple types of cancer and functions as an either oncogene or tumor suppressor (6-10). For instance, miR-190b upregulation modulated human Wilms' tumor cell growth, invasion, migration and apoptosis by targeting PTEN (6), and miR-190b expression was significantly enhanced in hormone-dependent breast cancer (7). Furthermore, miR-190b overexpression resulted in downregulation of insulin-like growth factor and results in poorer OS of patients with hepatocellular carcinoma (8). Contrary to these studies, miR-190b was demonstrated to exhibit tumor suppressive roles in osteosarcoma and gastric cancer $(9,10)$. miR-190b inhibited growth of osteosarcoma cells and induced apoptosis by regulating B-cell lymphoma-2 (9). In addition, miR-190b was found to be downregulated in radiotherapy-resistant gastric cancer cells, and miR-190b overexpression decreased cell viability and enhanced radio-sensitivity (10). However, there has been limited study of the role of miR-190b in CRC.

The aim of the present study was to determine the significance of miR-190b in CRC. miR-190b expression in CRC cell lines was compared with a normal colon epithelial cell line. Furthermore, we explored the role of miR-190b in regulating proliferation of CRC cells, colony formation and invasion, as well as the mechanism underlying its effects.

\section{Materials and methods}

Cell lines and cell culture. Human CRC cell lines HT29, SW480, SW620 and the normal colon epithelial cell line, FHC, were purchased from American Type Culture Collection. These cell lines were authenticated by ATCC using STR profiling and associated methods. The CRC cells were cultured in RPMI-1640 medium (Invitrogen; Thermo Fisher Scientific, Inc.), whereas the FHC cells were cultured in DMEM (Invitrogen; Thermo Fisher Scientific, Inc.) supplemented with $100 \mathrm{U} / \mathrm{ml}$ Penicillin, $50 \mathrm{mg} / \mathrm{ml}$ streptomycin, and 10\% FBS (all from Gibco; Thermo Fisher Scientific, Inc.) at $37^{\circ} \mathrm{C}$ with a humidified atmosphere containing $5 \% \mathrm{CO}_{2}$.

Cell transfection. miR-190b inhibitor (5'-AACCCAAUA UCAAACAUAUCA-3'), negative control (NC) for miR-190b inhibitor (NC-inhibitor; 5'-UUCUCCGAACGUGUC ACGU-3'), small interfering (si)RNA for forkhead box protein P2 (si-FOXP2; 5'-CGACAGAGACAAUAAGCAACA-3') and NC-siRNA (5'-GAAACCAAACGACGACAGUAA-3') were synthetized by Shanghai GeneChem Co., Ltd. pcDNA3.1 
containing an open reading frame of FOXP2 was purchased from GenScript. Transfection was performed by mixing Lipofectamine ${ }^{\circledR} 2000$ (Invitrogen; Thermo Fisher Scientific, Inc.) with synthesized miRNAs (50 nmol/1), siRNAs (20 nmol/l), or pFOXP2 (4 $\mu \mathrm{g})$ according to manufacturer's protocols. Cells were used for subsequent experiments after $48 \mathrm{~h}$ of transfection.

RNA isolation and reverse transcription-quantitative $P C R(R T-q P C R)$. Total RNA was extracted from cultured cells using TRIzol ${ }^{\circledR}$ reagent (Invitrogen; Thermo Fisher Scientific, Inc.) according to the manufacturer's protocol. miR-190b expression levels were detected using an ABI 7500 (Applied Biosystems Thermo Fisher Scientific, Inc.) using One Step TB Green ${ }^{\circledR}$ PrimeScript $^{\mathrm{TM}}$ RT-PCR Kit (Takara Bio, Inc.) after transcribing the extracted RNA into complementary DNA (cDNA) using MMLV Reverse Transcriptase First Strand cDNA Synthesis kit (Invitrogen; Thermo Fisher Scientific, Inc.) at $37^{\circ} \mathrm{C}$ for $15 \mathrm{~min}, 85^{\circ} \mathrm{C}$ for $5 \mathrm{~s}$, and $4^{\circ} \mathrm{C}$ for $60 \mathrm{~min}$. The sequences of the primers used were as follows: miR-190b forward, 5'-GGGTGATAT GTTTGATAT-3' and reverse, 5'-CAGTGCGTGTCGTGG AGT-3'; U6 small nuclear RNA forward, 5'-CTCGCTTCG GCAGCACA-3' and reverse, 5'-AACGCTTCACGAATT TGCGT-3'; FOXP2 forward, 5'-AACAACAGCAGGCTC TCCAG-3' and reverse, 5'-GGCACCTGCAGTGGTCTC-3'; and GAPDH forward, 5'-GGTGGTCTCCTCTGACTTCAA CA-3' and reverse, 5'-GTTGCTGTAGCCAAATTCGTT GT-3'. Expression levels of miR-190b or FOXP2 were calculated using the $2^{-\Delta \Delta C q}$ method with U6 snRNA or GAPDH as endogenous controls (11). Experiments were performed in triplicate. The thermocycling conditions were as follows: Initial denaturation at $95^{\circ} \mathrm{C}$ for $30 \mathrm{~s}$, followed by 40 cycles of $95^{\circ} \mathrm{C}$ for $10 \mathrm{~s}$ and $56^{\circ} \mathrm{C}$ for $30 \mathrm{~s}$.

Protein extraction and western blotting. Total protein was isolated from cultured cells using RIPA lysis buffer supplemented with protease inhibitor (both from Beyotime Institute of Biotechnology). Protein concentration was measured using a bicinchoninic acid quantitative detection reagent kit (Beyotime Institute of Biotechnology). Equal quantities $(50 \mu \mathrm{g})$ of protein samples were loaded on to a 10\% SDS gel and resolved using SDS-PAGE, and subsequently transferred to PVDF membranes (Beyotime Institute of Biotechnology). Membranes were blocked with non-fat milk at $4^{\circ} \mathrm{C}$ for $2 \mathrm{~h}$ and then incubated with primary antibodies (both supplied by Abcam) against FOXP2 (1:1,000, cat. no. ab207587) or GAPDH $\left(1: 1,000\right.$, cat. no. ab181602) at $4^{\circ} \mathrm{C}$ overnight. Membranes were then incubated with horseradish peroxidase-conjugated goat anti-rabbit secondary antibody (1:5,000, cat. no. ab6721; Abcam) at room temperature for $1 \mathrm{~h}$. Signals were developed using BeyoECL Star (Beyotime Institute of Biotechnology) according to the manufacturer's protocol. Experiments were performed in triplicate.

Cell Counting Kit-8 (CCK-8) assay. A CCK-8 assay was used to measure the cell proliferative capacity. A total of $3 \times 10^{3}$ cells/well were seeded in a 96 -well plate and cultured for $0,24,48$ or $72 \mathrm{~h}$ after seeding. CCK-8 reagent (Beyotime Institute of Biotechnology) was added to the plate and the cells were incubated for another $2 \mathrm{~h}$. Absorbance was measured at $450 \mathrm{~nm}$ using a Varioskan LUX multimode reader (Thermo Fisher Scientific, Inc.). Experiments were performed in triplicate.

Colony formation assay. A total of 300 cells/well were seeded in a 6-well plate and cultured for 2 weeks at $37^{\circ} \mathrm{C}$. Cell colonies were stained with crystal violet at room temperature for $30 \mathrm{~min}$ and the numbers of colonies in five randomly chosen fields were counted under an IX73 inverted microscope (Olympus Corporation) at a magnification of $\mathrm{x} 200$. Experiments were performed in triplicate.

Transwell invasion assay. A total of $1 \times 10^{5}$ cells in FBS-free medium were seeded into the upper chamber of Matrigel pre-coated inserts (Becton, Dickinson and Company). The lower chamber was filled with RPMI-1640 medium supplemented with $10 \%$ FBS. After 48 h of incubation, noninvasive cells were removed while the invasive cells were fixed with methanol at room temperature for $30 \mathrm{~min}$ and stained with $0.5 \%$ crystal violet at room temperature for $15 \mathrm{~min}$. Invasive cell numbers were counted under an IX73 inverted microscope at a magnification of $\mathrm{x} 200$ from five randomly chosen fields to assess the effects of miR-190b and FOXP2. Experiments were performed in triplicate.

Apoptosis analysis. Annexin V-fluorescein isothiocyanate/ propidium iodide (PI) Apoptosis Staining kit (Beyotime Institute of Biotechnology) was used to measure cell apoptosis according to the manufacturer's instructions. Cells were digested with $0.25 \%$ trypsin and stained with Annexin V and PI for $5 \mathrm{~min}$ in the dark at room temperature. Cell apoptosis was analyzed using a BD LSRFortessa flow cytometer (Becton, Dickinson and Company) with the equipped FACS Diva version 6.0 software (Becton, Dickinson and Company). Experiments were performed in triplicate.

Dual-luciferase activity reporter assay. TargetScan version 7.2 (targetscan.org/vert_72/) analysis was performed to determine the potential targets of miR-190b and a total of 223 genes were identified. Among these genes, FOXP2 was selected for following studies. Wild-type 3'-UTR of FOXP2 was amplified from the human CRC cell genome with the primers (Sense, 5'-GGTACCCTTTACAAACAGTTTTGACAG-3', and antisense, 5'-AAGCTTTGGTGTGAATGATGACTGG-3') and cloned into pGL3 luciferase vector (Promega Corporation) and termed pGL3-FOXP2-wt. A site-direct mutagenesis kit (Takara Bio, Inc.) was used mutate the FOXP2 3'-UTR using the primers (Fragment 1, forward 5'-CAGCGATCGCGAACT GACTTGTGAAACCTCAGCG-3', reverse, 5'-CTCGCAGTT ACTTCCAGTCCCTCAAAGCC-3'; and fragment 2, forward 5'-GTCTTTGGGTCATGATCAACGAACCGG-3' and reverse, 5'-TATGTTTAAACTTTATAAATGGGTCAAAAAGAATTA GA-3') and this was termed pGL3-FOXP2-mut. Cells were co-transfected with pGL3-FOXP2-mut or pGL3-FOXP2-wt combined with miR-190b inhibitor or NC-inhibitor using Lipofectamine ${ }^{\circledR}$ 2000. Luciferase activity was measured using a Dual-Luciferase reporter system (Promega Corporation) after $48 \mathrm{~h}$ of transfection. 
A 요

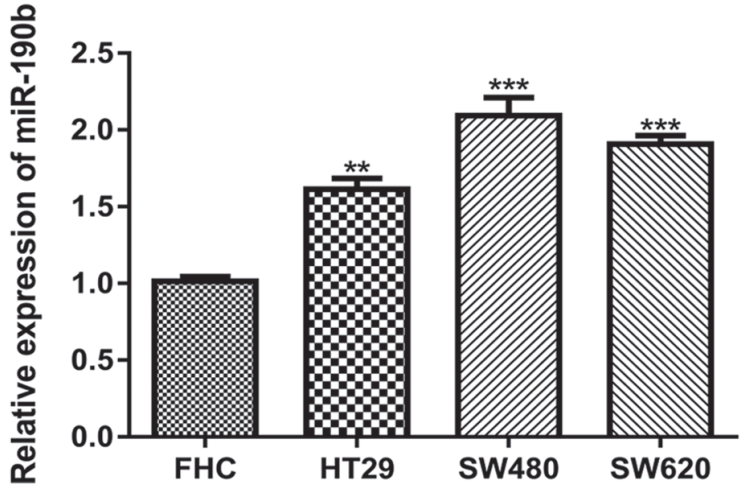

$\mathrm{B}$

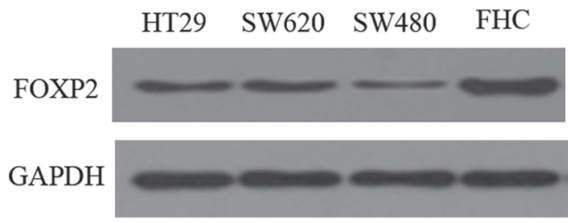

Figure 1. miR-190b expression is increased, whereas FOXP2 expression is decreased in CRC cell lines. (A) miR-190b expression and (B) FOXP2 expression was examined in HT29, SW480, SW620 cells and the normal colon epithelial cell line, FHC. ${ }^{* *} \mathrm{P}<0.01,{ }^{* * *} \mathrm{P}<0.001$ vs. FHC. CRC, colorectal cancer; miR-190b, microRNA-190b; FOXP2, forkhead box protein P2.
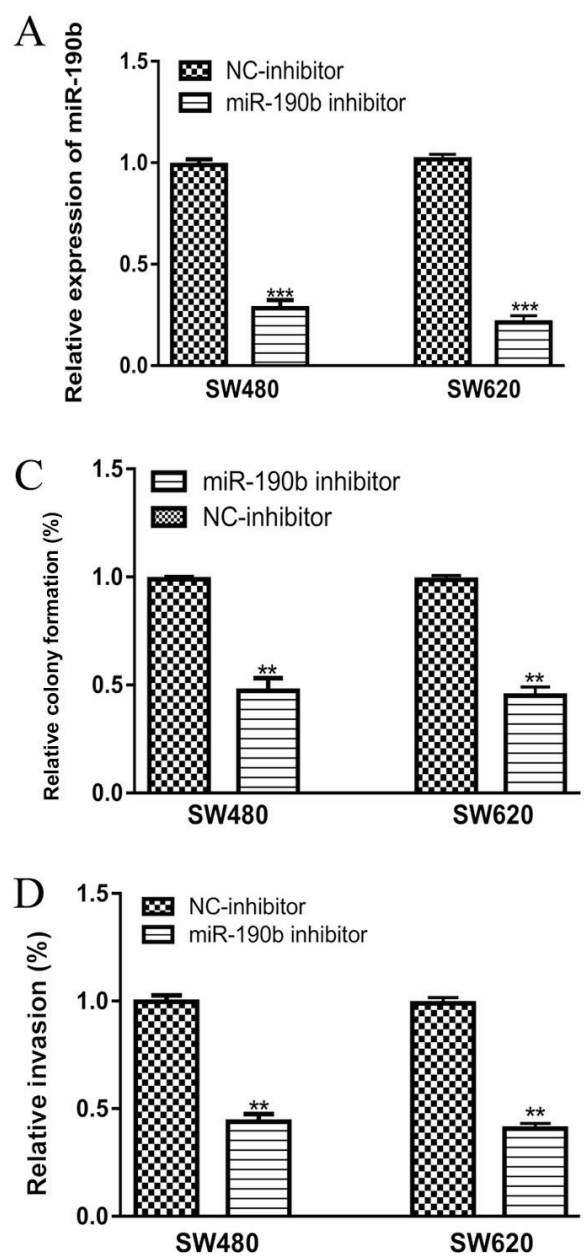
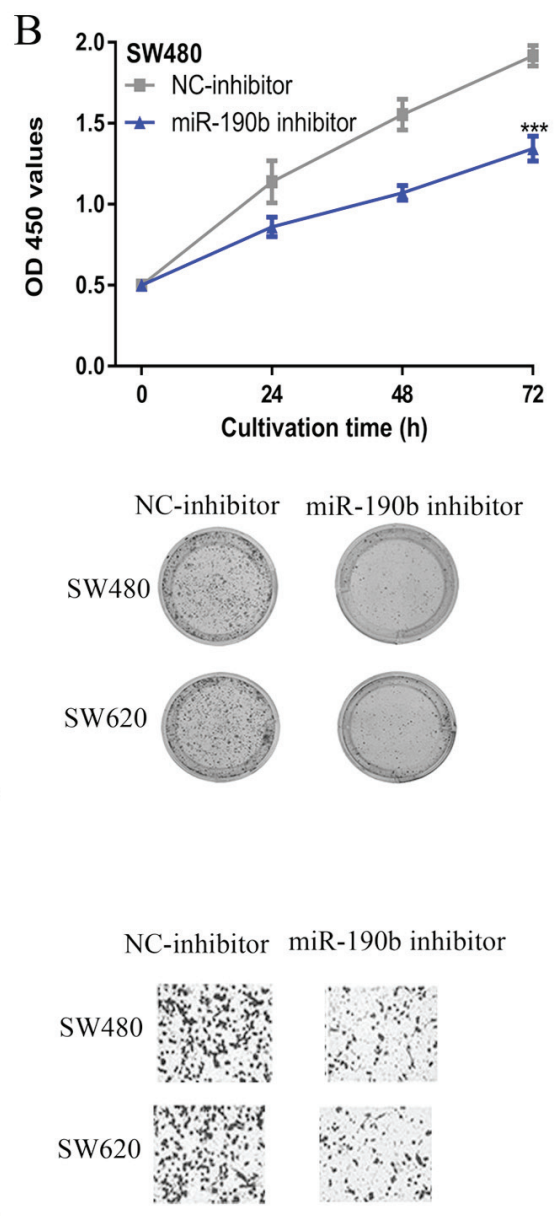

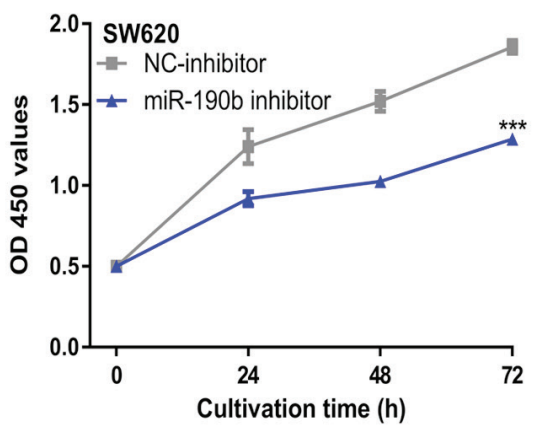

$\mathrm{E}$
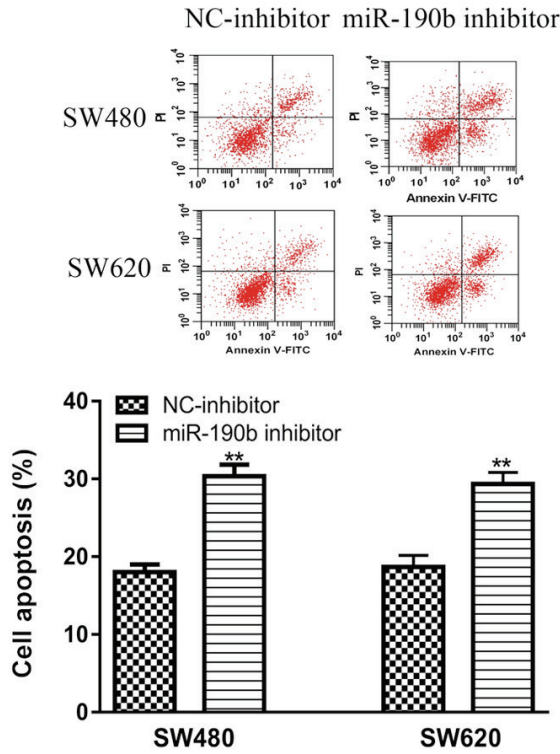

Figure 2. Downregulation of miR-190b reduces proliferation, colony formation and cell invasion of CRC cells. (A) miR-190b expression in cells transfected with miR-190b inhibitor was reduced compared with the same cells transfected with the NC-inhibitor. (B) Cell proliferation, (C) colony formation, (D) cell invasion (x200) and (E) cell apoptosis in CRC cells transfected with miR-190b inhibitor or NC-inhibitor. ${ }^{* *} \mathrm{P}<0.01,{ }^{* * * *} \mathrm{P}<0.001$ vs. respective $\mathrm{NC}$-inhibitor. CRC, colorectal cancer; miR-190b, microRNA-190b; NC, negative control; OD, optical density.

Statistical analysis. Statistical analysis was performed using GraphPad Prism version 6.0 (GraphPad Software Inc). Data are presented as the mean \pm standard deviation. Differences were analyzed using a Student's t-test for two groups or an ANOVA with a post hoc Tukey's test for $\geq 3$ groups. $\mathrm{P}<0.05$ was considered to indicate a statistically significant difference.

\section{Results}

miR-190b expression is upregulated, whereas FOXP2 expression is downregulated in CRC cell lines. miR-190b expression was significantly higher in the CRC cell lines compared with the FHC cell line (Fig. 1A). Conversely, FOXP2 expression 


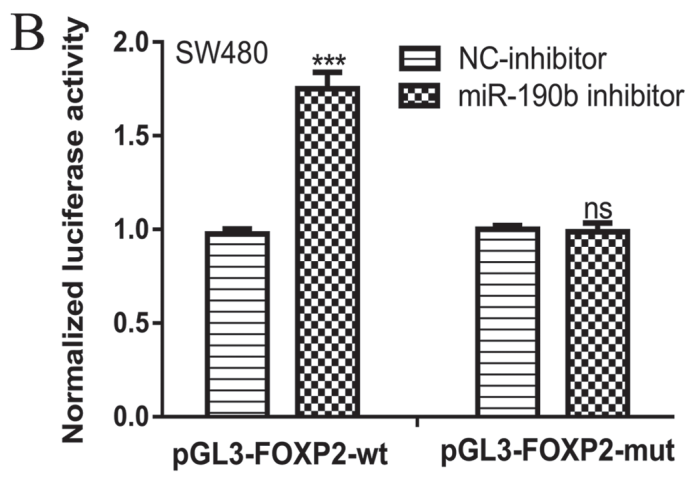

C

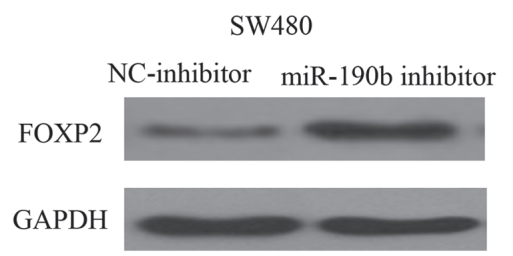

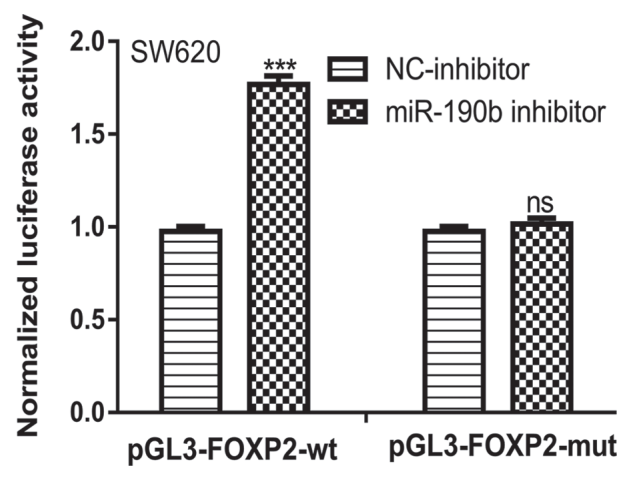

SW620

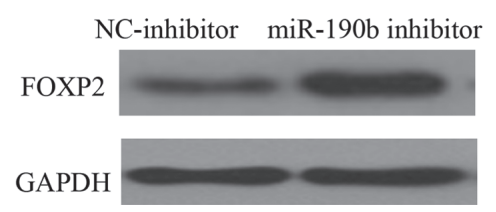

Figure 3. FOXP2 is a direct target of miR-190b. (A) A conserved binding site within the 3'-UTR of FOXP2 for miR-190b was identified. (B) Downregulation of miR-190b significantly increased the luciferase activity of pGL3-FOXP2-wt, but not pGL3-FOXP2-mut. (C) Downregulation of miR-190b appeared to increase the expression of FOXP2 in colorectal cancer cells. ${ }^{* * *} \mathrm{P}<0.001$ vs. respective NC inhibitor. miR-190b, microR NA-190b; FOXP2, forkhead box protein P2; UTR, untranslated region; wt, wild-type; mut, mutant; NC, negative control.

appeared to be lower in the CRC cell lines compared the FHC cell line as determined by western blotting (Fig. 1B). Therefore, the SW480 and SW620 cell lines were selected for the subsequent experiments as they have the highest and second-highest miR-190b expression level but lowest and second-lowest FOXP2 expression level.

Downregulation of miR-190b reduces cell proliferation, colony formation and cell invasion, and increases apoptosis. RT-qPCR analysis showed that transfection of the miR-190b inhibitor significantly decreased miR-190b levels compared with the NC-inhibitor (Fig. 2A). CCK-8 assay revealed that the knockdown of miR-190b was able to inhibit cell proliferation (Fig. 2B). Colony formation assay confirmed the results of CCK- 8 assay, which showed the introduction of miR-190b inhibitor has a long-term impact on cell proliferation (Fig. 2C). Transwell invasion showed cell invasion ability was inhibited by miR-190b inhibitor (Fig. 2D). Furthermore, flow cytometry showed cell apoptosis was enhanced by miR-190b inhibitor transfection, which further validated the results of CCK- 8 assay and clony formation assay (Fig. 2E).

FOXP2 is a direct target of miR-190b. TargetScan was used to identify candidate targets of miR-190b. A conserved binding site within the 3'-UTR of FOXP2 for miR-190b was identified (Fig. 3A). A luciferase activity reporter assay showed that transfection of the miR-190b inhibitor increased the luciferase activity of pGL3-FOXP2-wt, but not pGL3-FOXP2-mut (Fig. 3B). Western blotting showed that FOXP2 protein expression levels were enhanced by transfection of the miR-190b inhibitor in CRC cell lines (Fig. 3C).

FOXP2 is involved in miR-190b mediated cell behaviors. Transfection of si-FOXP2 transfection decreased both the mRNA and protein expression levels of FOXP2 in CRC cells (Fig. 4A and B). The effect of miR-190b inhibitor on FOXP2 expression was abolished by si-FOXP2 (Fig. 4A). FOXP2 downregulation enhanced $\mathrm{CRC}$ cell proliferation, colony formation and cell invasion, but decreased apoptosis compared with the NC-siRNA (Fig. 4C-F). Overexpression of FOXP2 decreased CRC cell proliferation, colony formation and cell invasion, but increased apoptosis compared with transfection with the pcDNA3.1 empty vector (Fig. 4C-F). In addition, rescue experiments revealed that the inhibitory effects of miR-190b inhibitor on CRC cell behaviors could be partially reversed by si-FOXP2 (Fig. 4C-F).

\section{Discussion}

Advancements in the understanding of CRC tumorigenesis have identified biomarkers to improve early diagnosis and molecular targeted therapies $(3,12)$. Numerous studies have highlighted the importance of cancer-associated miRNAs in the initiation and progression of CRC $(13,14)$. Li et al $(13)$ demonstrated that miR-452 was upregulated in CRC and its 

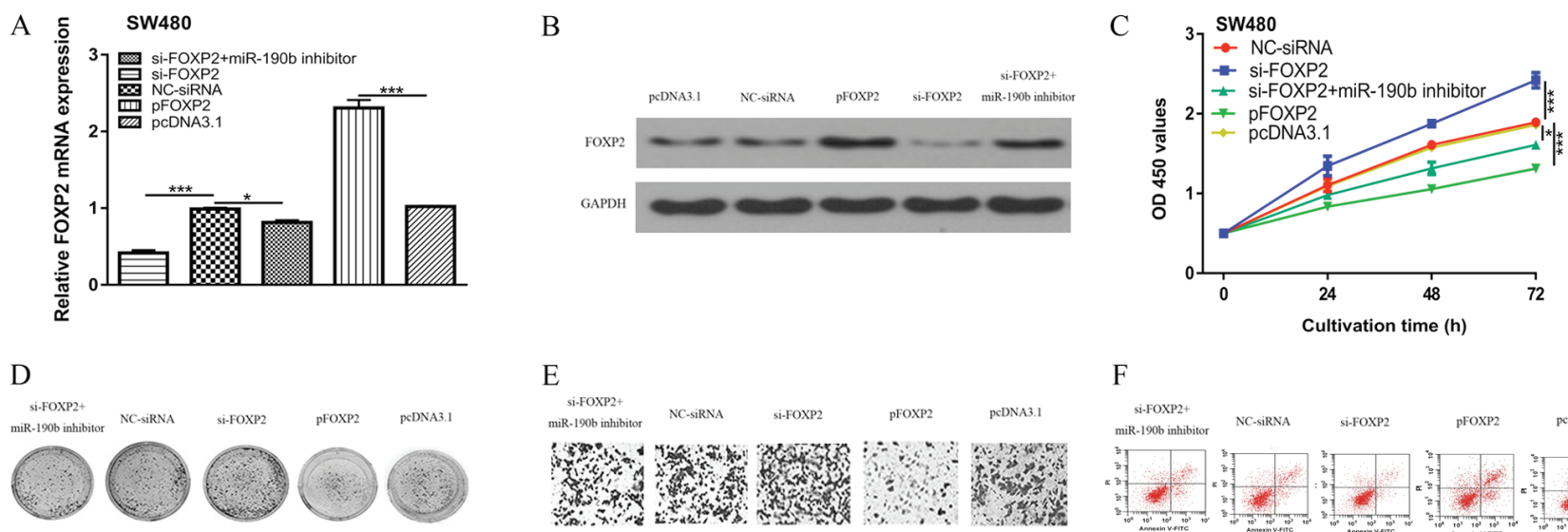

E
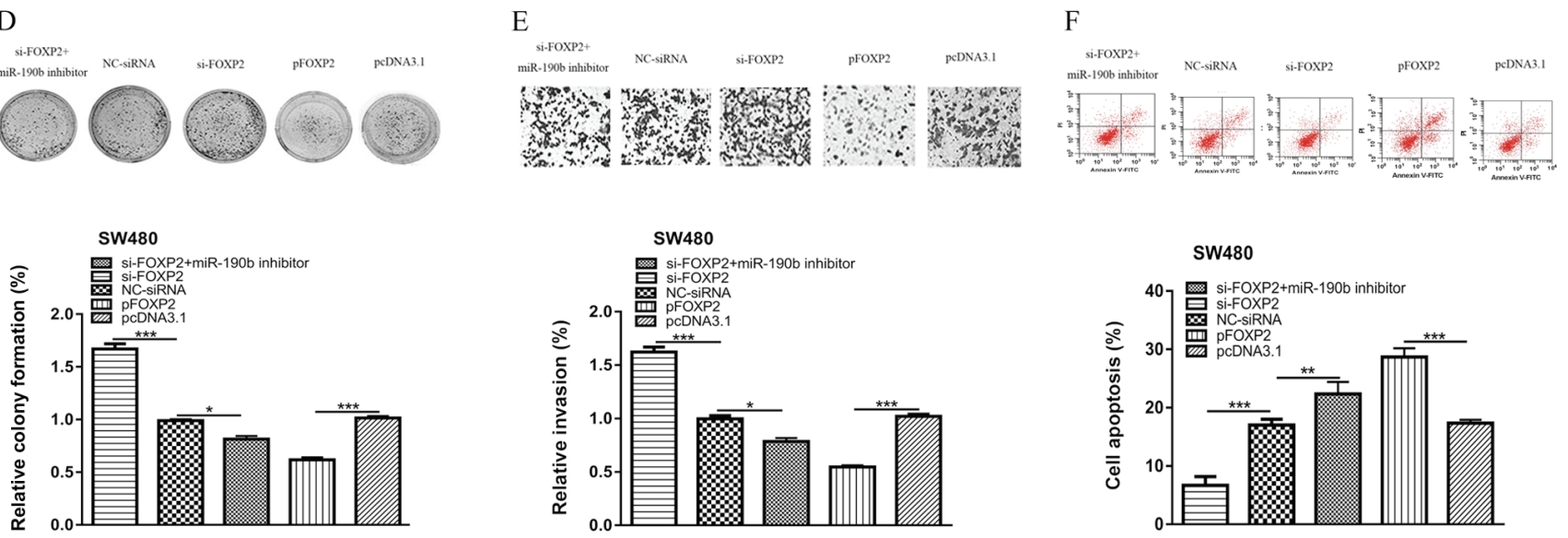

Figure 4. FOXP2 is involved in miR-190b-mediated alterations to CRC cell behavior. FOXP2 (A) mRNA and (B) protein expression levels were reduced in cells transfected with si-FOXP2, and increased in cells transfected with the FOXP2 overexpression vector. (C) Cell proliferation, (D) colony formation, (E) cell invasion (x200) and (F) cell apoptosis in colorectal cancer cell transfected with si-FOXP2, NC-siRNA, si-FOXP2 and miR-190b inhibitor, pcDNA3.1, or pFOXP2. ${ }^{*} \mathrm{P}<0.05,{ }^{* *} \mathrm{P}<0.01,{ }^{* * *} \mathrm{P}<0.001$. miR-190b, microRNA-190b; FOXP2, forkhead box protein P2; si, small interfering; NC, negative control.

upregulation activated the Wnt/ $\beta$-catenin signaling pathway, which promoted cancer progression in vitro and in vivo (13). Another study by Liu et al (14) revealed that miR-7702 acted as a tumor suppressor in CRC by targeting transcriptional adaptor 1 (14).

In the present study, miR-190b expression was found significantly upregulated in the assessed CRC cell lines compared with the normal colon epithelial cell line, FHC. Functional assays showed that downregulation of miR-190b reduced proliferation, colony formation and invasion of CRC cells, whilst increasing apoptosis in vitro. These results suggest that miR-190b functions as an oncogenic miRNA during progression of CRC, which is in consistent with its role in Wilms' tumor, breast cancer, and hepatocellular carcinoma (6-8). It is well documented that miRNAs function as tumor suppressors or oncogenes by regulating the expression of their specific target genes $(3,8-10,13,14)$. For example, genes including PTEN, IGF1, Bcl-2 have been identified as targets for miR-190b and involved in the tumor suppressive or oncogenic roles of miR-190b in cancers $(6,8-10)$. To identify the potential target of miR-190b, TargetScan bioinformatical analysis tool was employed, and we found FOXP2 was a putative target for miR-190b as it has previously been demonstrated to be abnormally expressed in a number of different types of cancer (15-17). FOXP2 downregulation promotes migration and invasion of breast cancer by influencing the tumor growth factor $\beta / \mathrm{SMAD}$ signaling pathway, indicating the tumor suppressive role of FOXP2 (15). FOXP2 was also found to be regulated by miRNAs including miR-196b and miR-23a in different types of cancer $(16,17)$. Here, FOXP2 was validated as a direct target of miR-190b through a luciferase activity reporter assay and western blotting. Knockdown of FOXP2 expression resulted in the stimulation effects on the malignancy behaviors associated with progression of CRC, whereas the reverse effects were observed when FOXP2 was overexpressed. These results indicated that FOXP2 functions as a tumor suppressor gene in CRC progression, which is in consistent with its roles reported in other cancer types (15-17). Additionally, rescue experiments showed that FOXP2 could reverse the inhibitory effects of miR-190b on CRC cells. miR-190b has opposing roles, dependent on the type of cancer (6-10), whereas FOXP2 functions as a tumor suppressor irrespective of the cancer (15-17). The results of the present study demonstrated that a miR-190b/FOXP2 axis contributed to the determination of cancer cell behavior. A limitation of the present study was the lack of in vivo studies to validate the conclusions of the in vitro studies. Animal models should be utilized to validate whether targeting the expression of miR-190b is an effective strategy for cancer treatment.

In summary, the present study demonstrated that miR-190b promoted proliferation, colony formation and cell invasion of CRC cells by negatively regulating the expression of FOXP2. The results demonstrated that miR-190b functioned as an oncogene in CRC. To the best of our knowledge, this is the first study to report the significance of miR-190b in CRC, and may thus lay a foundation to establish miR-190b as a therapeutic target for $\mathrm{CRC}$ in the future.

\section{Acknowledgements}

Not applicable. 


\section{Funding}

No funding was received.

\section{Availability of data and materials}

The datasets used/or analyzed during the current study are available from the corresponding author on reasonable request.

\section{Authors' contributions}

QZ, CL, QC, XL and CZ contributed to study design. QZ, CL, QC, XL, QW and CZ performed experiments and data analysis. QZ and CZ were major contributors in writing the manuscript. All author have read and approved the final manuscript.

\section{Ethics approval and consent to participate}

Not applicable.

\section{Patient consent for publication}

Not applicable.

\section{Competing interests}

The authors declare that they have no competing interests.

\section{References}

1. Siegel RL, Miller KD, Fedewa SA, Ahnen DJ, Meester RGS Barzi A and Jemal A: Colorectal cancer statistics, 2017. CA Cancer J Clin 67: 177-193, 2017.

2. Brenner H, Kloor M and Pox CP: Colorectal cancer. Lancet 383: 1490-1502, 2014

3. Yiu AJ and Yiu CY: Biomarkers in colorectal cancer. Anticancer Res 36: 1093-1102, 2016.

4. Bartel DP: MicroRNAs: Genomics, biogenesis, mechanism, and function. Cell 116: 281-297, 2004.
5. Lin S and Gregory RI: MicroRNA biogenesis pathways in cancer. Nat Rev Cancer 15: 321-333, 2015.

6. An NN, Shawn J, Peng JP, Wu MD and Huang LG: Up-regulation of miR-190b promoted growth, invasion, migration and inhibited apoptosis of Wilms' tumor cells by repressing the PTEN expression. Eur Rev Med Pharmacol Sci 22: 961-969, 2018.

7. Cizeron-Clairac G, Lallemand F, Vacher S, Lidereau R, Bieche I and Callens C: MiR-190b, the highest up-regulated miRNA in ER $\alpha$-positive compared to ER $\alpha$-negative breast tumors, a new biomarker in breast cancers? BMC Cancer 15: 499, 2015.

8. Hung TM, Ho CM, Liu YC, Lee JL, Liao YR, Wu YM Ho MC, Chen CH, Lai HS and Lee PH: Up-regulation of microRNA-190b plays a role for decreased IGF-1 that induces insulin resistance in human hepatocellular carcinoma. PLoS One 9: e89446, 2014

9. Kang M, Xia P, Hou T, Qi Z, Liao S and Yang X: MicroRNA-190b inhibits tumor cell proliferation and induces apoptosis by regulating Bcl-2 in U2OS osteosarcoma cells. Pharmazie 72: 279-282, 2017.

10. Wang C and Qiao C: MicroRNA-190b confers radio-sensitivity through negative regulation of Bcl-2 in gastric cancer cells. Biotechnol Lett 39: 485-490, 2017.

11. Livak KJ and Schmittgen TD: Analysis of relative gene expression data using real-time quantitative PCR and the 2(-Delta Delta C(T)) method. Methods 25: 402-408, 2001.

12. Lee JH, Hwang I, Kang YN, Choi IJ and Kim DK: Genetic characteristics of mitochondrial DNA was associated with colorectal carcinogenesis and its prognosis. PLoS One 10: e0118612, 2015.

13. Li T, Jian X, He H, Lai Q, Li X, Deng D, Liu T, Zhu J, Jiao H, Ye Y, et al: MiR-452 promotes an aggressive colorectal cancer phenotype by regulating a $\mathrm{Wnt} / \beta$-catenin positive feedback loop. J Exp Clin Cancer Res 37: 238, 2018.

14. Liu H, Li D, Fang H and Ning J: Species-specific function of microRNA-7702 in human colorectal cancer cells via targeting TADA1. Am J Transl Res 10: 2579-2589, 2018.

15. Chen MT, Sun HF, Li LD, Zhao Y, Yang LP, Gao SP and Jin W: Downregulation of FOXP2 promotes breast cancer migration and invasion through TGF//SMAD signaling pathway. Oncol Lett 15: 8582-8588, 2018.

16. Yu Z, Lin X, Tian M and Chang W: microRNA-196b promotes cell migration and invasion by targeting FOXP2 in hepatocellular carcinoma. Oncol Rep 39: 731-738, 2018.

17. Diao H, Ye Z and Qin R: miR-23a acts as an oncogene in pancreatic carcinoma by targeting FOXP2. J Investig Med 66: 676-683, 2018.

This work is licensed under a Creative Commons Attribution-NonCommercial-NoDerivatives 4.0 International (CC BY-NC-ND 4.0) License. 